\title{
Was den Wohnungsmarkt bewegt
}

\section{Integrierte Sozialplanung als Plattform für eine bessere Wohnqualität vor Ort}

\author{
ANDREAS STRUNK \\ Prof. Dr. phil. Andreas Strunk, Dipl. \\ Ing. ist Sozialpädagoge und Sozi- \\ alplaner. In der Gesellschaft für \\ Innovation, Systementwicklung \\ und Soziale Arbeit (GISAmbH) leitet \\ er Projekte der Organisationsent- \\ wicklung und Sozialforschung. \\ Weiterbildungen als Supervisor, lö- \\ sungsorientierter Berater und Case \\ Manager. Er ist Vorstandsmitglied \\ im Deutschen Berufsverband für So- \\ ziale Arbeit (DBSH), Landesverband \\ Baden-Württemberg. \\ prof.strunk@t-online.de
}

\author{
Soziale Projekte brauchen für ihre Wirksamkeit eine \\ integrierte Sozialplanung in den jeweiligen kommunalen \\ Kontexten. Diese muss einerseits für eine horizontale \\ Integration zwischen den beteiligten Ämtern und \\ den relevanten Gruppen sorgen und andererseits die \\ vertikale Integration im föderalen System zwischen \\ Region, Land, Bund, Europäischer Union verbessern.
}

Spätestens seit die »Bild-Zeitung « die »dramatische Kostenexplosion« bei Mieten und Nebenkosten entdeckt hat, wissen wir: Es droht eine neue Wohnungsnot in Deutschland (Ausgabe vom 13. Dezember 2012).

Der Grund ist darin zu suchen, dass die Wohnung ein doppelt gebundenes Gut ist. Einerseits dient sie zur Befriedigung eines zentralen Grundbedürfnisses des Menschen, andererseits unterliegt ihre Herstellung und Bewirtschaftung den Spielregeln des Marktes, was konkret heißt: Vermieter erwarten eine Rendite für ihr eingesetztes Kapital und Beiträge zur Refinanzierung ihrer »Vorhaltekosten«. Wohnungsbau und Wohnungsnutzung sind also aus strukturellen Gründen ständig in Bewegung. Wenn wir uns mit dem Thema »Wohnen in Bewegung « befassen, sollten wir uns anschauen, was den Wohnungsmarkt beeinflusst.

Zunächst fällt eine Dynamik ein, die als "Schweinezyklus « beschrieben wird: Der Wohnungsmarkt als Teil des Baumarktes lebt nicht von einem beständigen Gleichgewicht zwischen Nachfrage und Angebot. Er wird getrieben vom Auf und Ab zwischen Mangel und Überschuss. Zurzeit gibt es bundesweit wieder einen Wohnungsmangel. Nach Schätzungen des Bundesinstitutes für Bau-, Stadt- und Raumforschung fehlen aktuell in der Republik 265.000 Wohneinheiten. Der entsprechende Mangel wird vor allem in Wachstumsregionen deutlich. Das betrifft vor allem die Regionen um die Großstädte.
Hinzu kommt der Mangel an sogenannten Sozialwohnungen. Nach Aussagen des Pestel-Institutes in Hannover gibt es zurzeit 5,6 Millionen Haushalte, die über den Sozialen Wohnungsmarkt versorgt werden müssten; dort stehen aber zurzeit nur 1,6 Millionen gebundene Wohnungen zur Verfügung.

Wohnungen im freien Markt werden immer teurer; das wird deutlich sowohl an der Entwicklung der Bestandsmieten (in Stuttgart ab 2007 Erhöhung um $22 \%$ ) und an den Neuvermietungen (in Stuttgart ab 2007 Erhöhung um 20 \%). Für andere Städte ergibt sich ein ähnliches Bild. In vielen großstädtischen Regionen finden wir Anbietermärkte. Erfahrungsgemäß ist dort im Normalmarkt des Wohnens ein hoher Selektionsdruck nachweisbar. Haushalte mit eingeschränkter oder geringer Mietzahlungsfähigkeit oder mit Handicaps können in einem Subsidiärmarkt des Wohnens »entsorgt « werden (Heime, Provisorien, Fürsorgeunterkünfte, Asyle etc.). Diese Konstellation von »Selektion « und »Entsorgung « zwischen den beiden Systemen der Wohnungsversorgung scheint nur schwer überwindbar zu sein. Man kann sie beschreiben als die "Achterbahn des Wohnens«(vgl. Abb.). (1)

Nun kann man sich fragen, ob der derzeitige Zustand auf dem Wohnungsmarkt einem »Politikversagen « geschuldet werden muss. Dies ist nicht der Fall, denn in einer Phase des Nachfragermarktes, also in einer Zeit des tendenziellen regionalen Wohnungsleerstandes, 
war die Wohnungspolitik auf den drei relevanten Ebenen (Kommunen, Länder, Bund) vom Prinzip gekennzeichnet: »Was der Markt richten kann, soll von Politik nicht übernommen werden.«

Deregulierung und Privatisierung wurden Kennzeichen der Wohnungspolitik. Mithin bewusste Entscheidungen wurden getroffen. Sie waren ordnungspolitisch gekennzeichnet (»Freiheit des Marktes«) und finanzpolitisch getrieben (»Haushaltssanierung durch Wohnungsverkäufe und Unternehmensverkäufe«). Öffentliche Mittel für den Sozialen Wohnungsbau wurden darüber hinaus drastisch gekürzt. Auslaufende Bindungen führten zu einem immer stärker werdenden Verlust an gebundenen Wohnungen. Teilweise wurden von Wohnungsunternehmen bestehende Bindungen durch vorzeitige Rückzahlungen aufgelöst, weil die nun "frei« gewordenen Wohnungen am Markt rentierlicher platziert werden konnten und noch können.

In privatisierten Quartieren entsteht eine Dynamik dergestalt, dass Wohnungen saniert werden, die Mieten steigen und viele Haushalte diese Mieten nicht mehr zahlen können, der Zusammenhalt in der Bewohnerschaft wird geschwächt, die einsetzende Mobilität (ärmere Haushalte ziehen aus dem Quartier, zahlungskräftigere Haushalte bleiben oder ziehen ins Quartier), das gewachsene zivilgesellschaftliche Netzwerk beginnt zu zerfallen. (2)

An der geschilderten kollusiven Vernetzung der beiden Systeme Normal- markt und Subsidiärmarkt - der »Achterbahn des Wohnens " - sind die Agenten der Sozialen Arbeit beteiligt, wenn sie jenen Personen eine normale Wohnung mit der Begründung vorenthalten, dass sie nicht (oder noch nicht) »wohnfähig " sind. In diesem Zusammenhang sprach der Psychiater Klaus Dörner
Personen, die entweder an der Armutsgrenze oder darunter leben müssen. Insofern handelt es sich um einen besonderen Aspekt der Armut des Wohnens in einer Zeit der zunehmenden Spaltung zwischen »arm « und »reich «. Eine Spaltung, die schon lange beobachtet und analysiert wird. (3)

\section{"Gegen Wohnungslosigkeit hilft nur eins: eine Wohnung! "}

von »Heimträgern als Geiselnehmer«. Angesprochen wird die Strategie der Klientifizierung, die in vielen Bereichen der Sozialen Arbeit immer noch zur Hospitalisierung von Menschen führt. Aktuell ist das beispielsweise in der Behindertenhilfe im Zusammenhang mit den Bemühungen deutlich geworden, die Normalisierung des Wohnens für Behinderte voranzutreiben.

Aber nicht nur dort gibt es noch Beispiele der »Fehlplatzierung « durch Heimversorgung. Viele Einzelpersonen in der Altenhilfe, Wohnungslosenhilfe, Jugendhilfe und Straffälligenhilfe werden nicht als Nachfrager für den Normalmarkt des Wohnens betrachtet, weil sie in den relevanten Wohnraumbedarfsplanungen nicht als »Haushaltsnachfrage « berücksichtigt werden. Der Wohnungsmarkt reagiert nur auf Haushaltsnachfrage und nicht auf »Einzelfälle« in Heimen.

Das Schicksal der Vergessenen auf dem Wohnungsmarkt trifft in der Regel

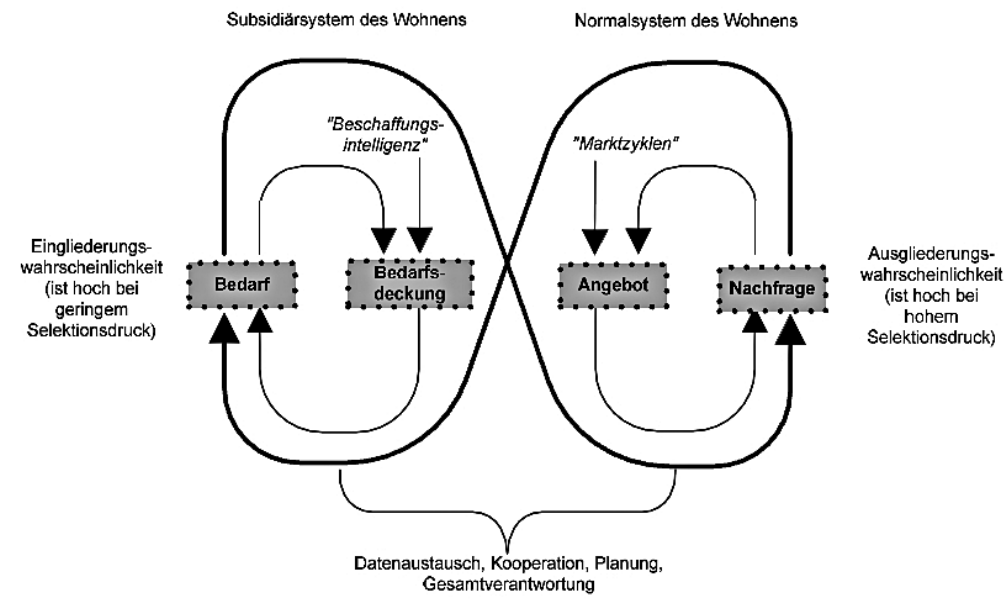

Mit dem Bild der "Achterbahn des Wohnens" kann die wechselseitige Abhängigkeit von Wohnungsangeboten im Bereich des Sozialmarktes und im Bereich des Wohnungsmarktes beschrieben werden.

Der von Klaus Dörner artikulierte Vorwurf der »Geiselnahme« spricht eine besondere Verantwortung für das Führungspersonal und die diagnostizierenden Fachkräfte der Sozialen Arbeit an. Heimunterbringung kann nur als Ultima-Ratio-Fall, als letzter Ausweg, in einem Interessenkonflikt akzeptiert werden.

In der Wohnungslosenhilfe gilt seit Mitte der 1970er Jahre zumindest für die verantwortungsvoll Handelnden der Satz: »Gegen Wohnungslosigkeit hilft nur eins: eine Wohnung!« Das hat beispielsweise in Stuttgart zur Erkenntnis geführt, dass im städtischen Wohnheim mindestens 60 Prozent der Neuzugänge mit normalem Wohnraum (entweder mit oder ohne Begleitung der Sozialarbeit) zu versorgen seien. (4) Das war der Start zur "Ambulantisierung « des Systems und zur Konzentration auf Strategien der Wohnungsbeschaffung.

In diesem Zusammenhang muss betont werden, dass Strategien der Normalisierung oder - wie es jetzt heißt der Inklusion nur möglich sind, wenn die Innovatorinnen und Innovatoren sich sorgfältig mit der kommunalen Sozialplanung abstimmen. Wir erleben zurzeit eine Regionalisierung der Wohnungspolitik. In diesem Kontext ist kommunale Sozialplanung ein zentraler Treiber. Denn nur was sich vor Ort bewährt, kann regionale Planung bedienen und nur was über die Region gelingt, kann Landespolitik überzeugen und nur über das Land kann die Bundespolitik beeinflusst werden. Gefordert ist also eine integrierte Sozialplanung im föderalen System.

Das hat auch die Bundesregierung erkannt, indem sie über das geltende Wohnraumförderungsgesetz (WoFG) in der Fassung aus dem Jahre 2002 Kommunen und Regionen auffordert, 
Wohnraumförderungskonzepte zu erarbeiten, indem sich alle Beteiligten und Betroffenen des Wohnungsmarktes besonders verständigen, wie vor allem die Versorgungslage von Haushalten, die Schwierigkeiten des »Marktzugangs « haben, verbessert werden kann. (5)

Wohnen bietet eine Basisfunktion in der Gemeinde. Auf kommunaler Ebene wird zunehmend erkannt, dass Deregulierung und Privatisierung im Wohnungsmarkt vor Ort erhebliche Nachteile gebracht hat. Insofern scheint die Polarisierung zwischen Markt und Staat überwunden zu sein. Eine Versachlichung über die zukünftige Gestaltung der Wohnungspolitik ist zu erwarten. Argumente wie die des ehemaligen Richters am Verfassungsgericht Siegfried Broß (6) werden diskutiert: Es sei an der Zeit, einige Privatisierungen rückgängig zu machen, indem wieder stärker nachhaltige Planungsperspektiven entwickelt werden, die Architektur des Steuersystems zugunsten einer besseren Ausstattung des kommunalen Sektors verändert wird und »Rekommunalisierungsfonds" aufgebaut werden zum Rückkauf relevanter Basisangebote.

Soziale Überlegungen und Projekte bedürfen - wenn sie sich weiter ausbreiten sollen - einer integrierten Sozialplanung in den jeweiligen kommunalen Kontexten. Diese hat eine komplexe Aufgabenstellung: Einerseits hat sie eine horizontale Integration (zwischen den beteiligten Ämtern und den relevanten Share- und Stakeholdern) zu leisten und andererseits die notwendige vertikale Integration im föderalen System (Region, Land, Bund, EU) zu verbessern. (7)

Aus der Sicht von innovativen Vorhaben der Zivilgesellschaft (z. B. neue Wohnformen) oder der Sozialen Arbeit (z. B. Inklusionsprojekte) geht es um die Aktivierung kommunaler Potenziale, die für Planung und Management kommunaler Wohnungspolitik relevant sind. Hier gibt es entsprechende Aufgaben der Einmischung. Relevante Themen für die Einmischung können u. a. sein (8): Flächennutzung, Grundstücksverkehr, Wohnungsneubau, Wohnungsbestandspflege, Wohnungsbelegung, Wohnungsvermietung, Wohnungskaufkraft, Quartiersentwicklung, neue Wohnformen, Konversionsmanagement, Beteiligungsmanagement, Entsorgungsdynamik, Ämterentwicklung, Politikentwicklung.
Die integrierte Sozialplanung kann den Rahmen bilden für die Selbstorganisation von Initiativen auf dem Weg zur Verbesserung von Wohnqualität vor Ort. Das wäre ein wechselseitiger Bedingungszusammenhang, der vielen Orten des Wohnens zu wünschen ist. Das Ziel wäre, die »Alltagstauglichkeit unserer Städte « zu erhöhen. (9)

\section{Anmerkungen}

(1) Die "Achterbahn des Wohnens" wurde vom Autor zum ersten Mal im Jahre 1979 vorgestellt. Vgl. dazu: Andreas Strunk, Wohnungspolitik für Nichtsesshafte, in: Gefährdetenhilfe 4 (1979), S. 29 ff.

(2) Vgl. dazu: Der Spiegel 4 (2013), S. 36 f. Zum Höchstgebot: Im Wahlkampf setzt Kanzlerkandidat Peer Steinbrück auf das Thema steigende Mieten. Als Finanzminister verkaufte er dagegen noch komplette Wohnsiedlungen an Investoren.

(3) Vgl. dazu: Thomas Specht, Spaltung im Wohnungsmarkt - die unsichtbare Armut des Wohnens, in: Dieter Döring, Walter Hanesch und ErnstUlrich Huster, Armut im Wohlstand, Frankfurt am Main 1990, S. 227-243.

(4) Vgl. dazu: Andreas Strunk, Ambulante Betreuung, in: Gefährdetenhilfe 4 (1976), S. 4-7.

(5) Vgl. dazu: Andreas Strunk, Kooperationsverträge nach dem $\mathrm{WoFG}$ - Instrumente der Wohnungssicherung, in: NDV 11 (2003), S. 478-483.

(6) Vgl. Siegfried Broß u. a., Kommunen für den Bürger, in: Frankfurter Rundschau vom 26.10.2012.

(7) Dies wird näher ausgeführt in einem Positionspapier des Vereins für Sozialplanung (VSOP) zum Thema Wohnen. Das Positionspapier wird auf der Jahrestagung 2013 des VSOP in Bremen vorgestellt (25./26.4.2013).

(8) Vgl. dazu: Andreas Strunk, Planung und Management kommunaler Wohnungspolitik, in: Wohnungspolitik in Zeiten knapper Kassen, Berlin 2003, S. 44-75.

(9) Vgl. dazu: Andreas Feldtkeller, zur Alltagstauglichkeit unserer Städte. Wechselwirkungen zwischen Städtebau und täglichem Handeln, Berlin/Tübingen 2012.

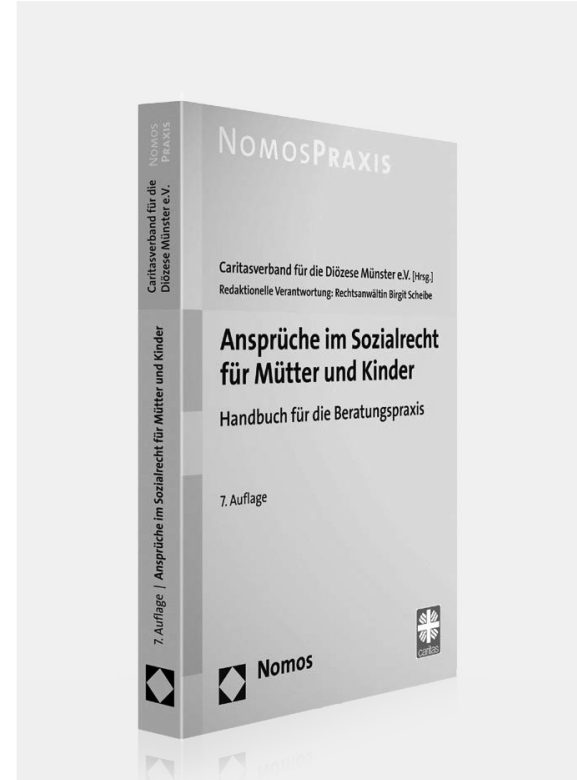

\section{Ansprüche im Sozialrecht} für Mütter und Kinder

Handbuch für die Beratungspraxis

Herausgegeben vom Caritasverband für die Diözese Münster e.V.

Redaktionelle Verantwortung Rechtsanwältin Birgit Scheibe

7. Auflage 2012, 451 S., brosch., 39,-€, ISBN 978-3-8329-5969-2

Auf aktuellem Stand und natürlich unter Berücksichtigung der Änderungen durch die Hartz-IVReform sowie beim Elterngeld und in der Krankenversicherung bietet die Neuauflage allen Sozialrechtsberatern/-innen systematisch wie praxisorientiert Lösungen zu Fragen der juristischen Beratungspraxis. Dabei werden auch wichtige Fragen zum SGB III, MuSchG und Krankenschutz erörtert. Die notwendigen Bezüge zum UVG, Kindergeld und Kinderzuschlag werden hergestellt und Fragestellungen aus der Jugendhilfe und zum BAföG nebst den familienrechtlichen Aspekten aufgegriffen.

www.nomos-shop.de/12910

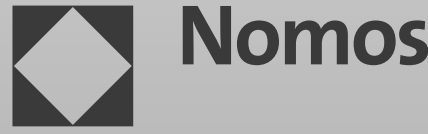

